\title{
O tema escolhido pela presidência da RGS-IBG 2017
}

Descolonizando os conhecimentos geográficos ou reproduzindo a colonialidade?

The 2017 RGS-IBG chair's theme: decolonising geographical knowledges, or reproducing coloniality?

RGS-IBG 2017 : décolonisation des connaissances géographiques ou réproduction de la colonialité ? Thème présidentielle

El tema escogido por la presidencia de la RGS-IBG 2007: ¿Descolonizando los conocimientos geográficos o reproduciendo la colonialidad?

\section{James Esson, Patricia Noxolo, Richard Baxter, Patricia Daley e Margaret Byron}

Tradutor. Katielle Susane do Nascimento Silva

\section{OpenEdition}

Journals

\section{Edição electrónica}

URL: https://journals.openedition.org/terrabrasilis/8805

DOI: $10.4000 /$ terrabrasilis. 8805

ISSN: 2316-7793

\section{Editora}

Rede Brasileira de História da Geografia e Geografia Histórica

\section{Refêrencia eletrónica}

James Esson, Patricia Noxolo, Richard Baxter, Patricia Daley e Margaret Byron, «O tema escolhido pela presidência da RGS-IBG 2017», Terra Brasilis [Online], 15 | 2021, posto online no dia 31 julho 2021, consultado o 05 dezembro 2022. URL: http://journals.openedition.org/terrabrasilis/8805 ; DOI: https:// doi.org/10.4000/terrabrasilis.8805

Este documento foi criado de forma automática no dia 5 dezembro 2022.

All rights reserved 


\section{O tema escolhido pela presidência da RGS-IBG 2017}

Descolonizando os conhecimentos geográficos ou reproduzindo a colonialidade?

The 2017 RGS-IBG chair's theme: decolonising geographical knowledges, or reproducing coloniality?

RGS-IBG 2017 : décolonisation des connaissances géographiques ou réproduction de la colonialité ? Thème présidentielle

El tema escogido por la presidencia de la RGS-IBG 2007: ¿Descolonizando los conocimientos geográficos o reproduciendo la colonialidad?

James Esson, Patricia Noxolo, Richard Baxter, Patricia Daley e Margaret Byron

Tradução : Katielle Susane do Nascimento Silva

\section{NOTA DO EDITOR}

A versão original desse artigo foi publicada por James Esson, Patricia Noxolo, Richard Baxter e Margaret Byron na revista Area, Volume 49, Issue3, September 2017, Pages 384-388. Disponível em https://doi.org/10.1111/area.12371. Tradução da Dra. Katielle Susane do Nascimento Silva.

Queremos agradecer a todos os membros do grupo de trabalho RACE, bem como a todos os outros colegas não brancos e indígenas, amigos e ativistas que dialogaram conosco na preparação deste breve comentário. Gostaríamos de agradecer aos editores Peter Kraftl e Kavita Datta pelo apoio. 


\section{Introdução}

1 O tema da plenária escolhido pela presidência da Conferência Anual da Royal Geographical Society (com o Institute of British Geographers) (RGS-IBG), de 2017, é "Descolonizando o conhecimento geográfico: abrindo a geografia para o mundo". De acordo com sumário orientador da conferência, o evento constituirá parte de uma agenda para

questionar implicitamente as reivindicações universais de conhecimentos associados ao Ocidente, e interrogar ainda mais como tais conhecimentos continuam a marginalizar e desconsiderar lugares, pessoas e conhecimentos em todo o mundo. (RGSIBG, 2017: n.p.)

2 Neste documento, objetivamos explicar por que essa busca de uma consciência crítica através de uma abordagem descolonial pode fazer mais mal do que bem, numa disciplina que pode não estar pronta ou mesmo não ser capaz de responder o desafio da descolonização. Para ser claro, este comentário assinala o confronto de questões estruturais dentro da disciplina. Nós não estamos procurando difamar ou desacreditar indivíduos ou grupos que estão lutando por justiça social e que têm dedicado as suas carreiras para educarem-se a si próprios e aos outros para serem críticos do colonialismo e colonialidade. Por colonialidade, entendemos

padrões de poder instituído a longo prazo que emergiram como resultado do colonialismo, mas que definem a cultura, o trabalho, as relações intersubjectivas e a produção de conhecimento muito para além dos limites estritos das administrações coloniais. (Maldonaldo-Torres, 2007: 243)

Estamos intervindo nesta conjuntura porque a descolonização implica a eliminação do domínio colonial em curso, conectando assim movimentos para desmantelar a classificação social racista da população mundial sob poder do mundo eurocêntrico (ver Mignolo, 2008; NdlovuGatsheni, 2015), com as demandas de líderes indígenas por uma reestruturação radical das terras, recursos e riqueza a nível global (ver Tuck e Yang, 2012). O foco na descolonização do conhecimento deveria ser posicionado como um meio para este fim, e não como um fim em si mesmo. Argumentamos que a ênfase atual na descolonização do conhecimento geográfico mais do que nas estruturas, instituições e práxis, e a frase dissimulada "abrindo a geografia para o mundo" (como se os geógrafos, e de facto a Royal Geographical Society (RGS), nunca tivessem envolvidos na exploração, colonização e expropriação contínua do mundo e dos seus recursos) dilui o potencial transformador da descolonização e descolonialidade, enquanto oculta estruturas opressivas na disciplina e recentra os grupos não-indígenas, brancos e privilegiados na arquitetura global da produção do conhecimento. Para evitar isto, a disciplina precisa assegurar que os termos, a partir dos quais, os geógrafos iniciam os debates sobre descolonização e descolonialidade sejam determinados por aqueles que estão à margem, os quais têm sido racializados como indígenas e não brancos pela colonialidade.

\section{A descolonização não é uma metáfora}

A descolonização é fundamentalmente 'inquietante', tem uma ressonância peculiar nas antigas colônias (Tuck e Yang, 2012), mas em termos globais a descolonização procura derrubar a colonialidade do poder e a sua matriz constitutiva (Quijano, 2000: 533). Tem 
existido movimentos descoloniais organizados por ativistas no Reino Unido e em todo o mundo há algum tempo. Por conseguinte, descolonização é uma mudança política radical que não pertence à academia, e não é um "tema" que pode ser abordado durante três dias de conferência e, em seguida, ser deixado de lado. Por isso, embora reconheçamos que o cultivo da consciência crítica, como articulado na chamada da conferência "descolonizar o conhecimento geográfico" seja importante, nós propomos que a consciência crítica não pode ser o foco de genuínos movimentos descoloniais, pois sem ativismo não enfrentaremos o imperialista-supremacista branco-patriarcalcapitalista (Hooks, 2004) nem alcançaremos uma reestruturação radical da terra, dos recursos e da riqueza a nível global. Há quem leve estes pontos mais longe, e argumente que a construção da consciência crítica levada adiante por parte dos acadêmicos mina e dilui os movimentos descoloniais, como é o excelente exemplo daquilo a que Tuck e Yang (2012) chamam de "movimentos para a inocência": estratégias e posicionamentos que visam aliviar os sentimentos de culpa ou cumplicidade daqueles que se beneficiam da colonialidade, sem terem que alterar de todo a sua posição privilegiada.

Um primeiro passo fundamental para evitar que a conferência RGS-IBG se torne um movimento coletivo para a inocência será os geógrafos aceitarem o assinalar de Rivera Cusicanqui para uma "prática de descolonização" ativa (2012: 100) em trabalhos acadêmicos que alcancem mais profundidade do que o vocabulário. Por quê? Porque "as nossas instituições devem ser submetidas a um processo de descolonização tanto do conhecimento como da universidade como instituição" (Mbembe, 2016: 11). Prevemos que isto aconteça como parte de uma agenda descolonial que se afasta de uma fixação com epistemologia, e em direção à práxis que tanto revela e busca endereçar, quais formas de violência e 'microagressões' experienciadas pelos grupos indígenas e racializados dentro da academia e na vida quotidiana, são normalizadas e oficialmente sancionadas por disposições institucionais (ver também Mbembe, 2016; Tate, 2014; Tejeda et al., 2003; Todd, 2016). Na academia especificamente, Mahtani (2014) argumenta que as hierarquias racializadas produzem "espaços materiais emocionalmente tóxicos" para os geógrafos não-brancos e indígenas, os quais são invisibilizados pelo discurso liberal. Fundamentalmente, dada a composição demográfica da disciplina, ter em conta a invocação de Rivera Cusicanqui exigiria que os geógrafos confrontassem a supremacia branca, o privilégio branco, e o racismo no passado e no presente. Isto porque a interseccionalidade da indigeneidade e raça, e a adoção de um ponto de vista antirracista são proeminentes no trabalho pioneiro de estudiosos descoloniais (ver Aime Cesaire, Frantz Fanon, Walter Mignolo, Anibal Quijano, Ngugi wa Thiong'o e Sylvia Wynter).

6 Este ponto sobre o confronto da supremacia branca, privilégio branco e o racismo é particularmente relevante porque é notável que nem a raça, nem a indigeneidade (para além de uma ampla referência aos 'saberes indígenas') foram mencionadas no resumo original da conferência de 2017. Registamos que os ajustes subsequentes na página web da conferência e a demografia dos oradores da plenária vieram logo após intervenções de geógrafos não brancos e indígenas. Da mesma forma, o resumo original, a página web da conferência e numerosos convites para sessões não conseguiram incentivar uma reflexão substantiva sobre a implicação da disciplina no estabelecimento histórico do colonialismo e a reprodução da colonialidade nos dias de hoje. A descolonização do conhecimento geográfico não pode ocorrer enquanto estruturas racistas e colonialistas herdadas de disciplina colonial e imperialista do passado se mantiverem. Assim, 
apelamos aos geógrafos, e instituições geográficas chave, tal como, o RGS-IBG, a reconhecerem estruturas racializadas opressivas na disciplina e demonstrarem um compromisso com o antirracismo como pré-requisito para embarcar em movimentos descoloniais dentro da disciplina. Esta tem de ser uma característica óbvia pelas razões acima mencionadas, mas também porque a circulação social da raça como força social é frequentemente apoiada por ficções raciais bem-intencionadas, mantidas pelo grupo racial dominante, incluindo aqueles que se veem a si próprios como liberais progressistas. Uma destas ficções é que se uma pessoa não tem opiniões racistas, como definidas normativamente, então não está envolvida na perpetuação da desigualdade racial e, portanto, não tem que adotar uma posição explicitamente antirracista (Bonilla Silva citada em Walter e Butler, 2013).

7 Para ser claro, não estamos pedindo que seja oferecida aos acadêmicos e ativistas racializados como não brancos e indígenas uma participação socialmente mais equitativa nas estruturas colonialistas e racistas de dominação e exploração existentes (tal movimento, como Tuck e Yang (2012: 28), assinalaram, é incomensurável com a descolonização). Insistimos que o desmantelamento destas estruturas seja uma característica explícita de qualquer agenda descolonial estabelecida na conferência ou em torno da mesma. Na secção que se segue, explicamos por que razão o não envolvimento com as ideias apresentadas acima e a não resposta às nossas preocupações sobre a conferência reproduzirá a colonialidade dentro da disciplina.

\section{Reproduzindo colonialidade?}

8 Mais imediatamente, é necessária informação sobre os movimentos descoloniais mais a longo prazo que já tiveram e terão lugar dentro da geografia britânica: compromissos práticos com e por múltiplos atores descoloniais precisam ser bem divulgados antes, durante e depois da conferência. Sem esta informação, o grupo de acadêmicos e ativistas indígenas e não brancos continuará a ver a próxima conferência da RGS-IBG com um certo grau de ceticismo. Há duas razões chave para isso. Primeiro, embora o resumo afirme que "debates em torno dos conhecimentos geográficos decolonizantes têm vindo a ser crescentemente importante entre professores ativistas e acadêmicos na última década" (RGS-IBG, 2017: $\mathrm{np}$ ), a maioria dos geógrafos tem permanecido periférica às lutas descoloniais contemporâneas, incluindo casos de grande visibilidade como as campanhas Rhodes Must Fall em Oxford e na África do Sul, mas também no conjunto vibrante de atividades que acontecem além da academia aqui no Reino Unido. Um exemplo deste último foi o Decoloniality London, que estabeleceu um programa de ensino sobre o pensamento e práxis descoloniais fora do ensino superior. Em segundo lugar, o estudo da descolonização dentro da geografia e disciplinas aliadas não ocorre fora da política da academia, onde alguns têm a ganhar como guardiães e como vozes privilegiadas. $O$ papel da branquitude nestas normas e práticas precisa ser interrogado, uma vez que estudiosos indígenas e os racializados como não brancos já estão sujeitos à colonialidade e lutam para manter uma presença dentro da disciplina (ver Todd, 2016; Peake e Kobayashi, 2002).

9 As preocupações acima ressoam com a assertiva de Rivera Cusicanqui (2012: 95-7) de que, em poderosos contextos acadêmicos dominado por estudiosos ocidentais não indígenas e brancos, onde pessoas indígenas (e, por conseguinte, os não brancos) não são centrais e não ditam as regras, uma "versão logocêntrica e nominalista" das 
abordagens descoloniais é recirculada e comercializada (Rivera Cusicanqui, 2012: 102). Tuck e Yang (2012) reiteram este ponto, e notam como a recente proliferação da linguagem descolonial por acadêmicos não indígenas e brancos pode reproduzir a colonialidade. Isto porque reforça as estruturas da supremacia branca que reafirma o privilégio branco. Por privilégio branco entendemos "vantagem, em grande parte, não expressa, que se acumula por um sistema estrutural social normalizado com base nas experiências, valores, cultura e percepções do povo Branco" (Walter and Butler, 2013: 401). Todd ilustra estes pontos descrevendo como na academia,

Os corpos indígenas, histórias, conhecimentos e 'contatos' ('informadores', 'participantes' ou 'interlocutores') atuam como uma espécie de moeda ou capital que se concentra nas mãos de estudiosos e administradores não indígenas. Por conseguinte, na sua esmagadora maioria, são ainda os brancos que controlam o fluxo deste conhecimento e os parâmetros destas relações. (2017: n.p.)

Para ser claro, ainda é possível e talvez até necessário falar sobre as visões de mundo indígenas a partir de "fora", e engajar no diálogo, mas sem uma base de poder indígena e não-branco há um risco real de que, "a descolonização se torne uma indústria domesticada de ideias" (Sium et al., 2012: IV) que é removida da lógica situada do ativismo e do conhecimento indígena e não-brancos. Além disso, a hierarquia colonialista de superioridade racial principalmente branca e a inferioridade de indígenas e não brancos, é invisibilizada e passa desapercebida. Isto cria ainda mais um cenário em que, ao procurar "descolonizar o conhecimento geográfico e abrir a geografia para o mundo", os geógrafos correm o risco de falar não por, mas ao invés daqueles não apenas dispostos e capazes, mas também ansiosos e equipados, para falar por si próprios (Bob Geldof, recentemente galardoado com uma medalha de Patrono do RGS-IBG, é um exemplo contemporâneo disto em ação; ver Daley [2005] para uma discussão detalhada). Existe, portanto, um perigo real de que a conferência conduzirá a uma maior subjugação, uma vez que alguns estudiosos indígenas e não brancos pretendem evitar uma reunião que ignora e reproduz a colonialidade, enquanto aqueles que estão a planejar participar são marginalizados e sentem que correm o risco de serem cooptados. Alguns geógrafos não brancos e indígenas já foram marginalizados no período que antecedeu a conferência e não se sentiram capazes de contribuir para a organização do evento. É, portanto, imperativo que os organizadores da conferência, o RGS-IBG e os participantes considerem como irão abordar a opressão adicional dos acadêmicos indígenas e não brancos, e mitigar a questão tripla da cooptação, da desresponsabilização do pensamento descolonial e reafirmação do privilégio branco que pode ocorrer por causa da conferência.

\section{Conclusões}

11 A decolonização é um desafio radical para 'desestabilizar' a arquitetura de privilégio (Tuck, e Yang 2012: 3). Deve envolver a descolonização da mente e a ação revolucionária (Fanon, 1967). A primeira conferência RGS-IBG sobre descolonização proporcionou uma oportunidade de estabelecer a geografia como uma disciplina mais progressiva. Em vez disso, a conferência refletiu problemas estruturais contínuos de raça e indigeneidade no ensino superior e na sociedade em geral. Um movimento descolonial efetivo em geografia precisa focar na revelação dos problemas da colonialidade dentro e fora da disciplina, e atentar para desestabilizar a arquitetura do privilégio branco-patriarcal (ver Shilliam, 2015). Iniciar debates sobre descolonização e 
descolonialidade com os racializados como indígenas e não-brancos é um ponto de partida fundamental para uma tal mudança. Para este fim, recomendamos que durante a conferência e nos meses seguintes tanto os geógrafos como as principais instituições geográficas, como o RGS-IBG, se empenhem numa verdadeira reflexão sobre o (não) estatuto de raça e indigeneidade na disciplina. Isto deveria resultar em mudanças que interrompessem os sistemas de privilégios estabelecidos, por exemplo em relação ao tema deste manuscrito, à política de nomeação de presidentes, eleição e à organização da conferência.

Ao escolher este tema em particular, o RGS-IBG posicionou a geografia dentro de uma agenda radical em torno da decolonização como conhecimento e prática. Os autores deste comentário estão tentando manter o otimismo de que a conferência de 2017 fornecerá evidências tangíveis de que a disciplina está de facto pronta, e capaz de, responder ao desafio da descolonização. Este sentimento de otimismo não deve ser lido como um anseio passivo por um futuro abstrato idílico, mas sim como um sinal do nosso descontentamento no presente, como articulado acima, ao lado da crença

nas possibilidades criativas que se tornam disponíveis quando nos reconhecemos uns aos outros (e cada 'Outro') à medida que nos reunimos com base num compromisso de descolonização. (Gaztambide-Ferneez, 2012: 61, ênfase no original)

Resta saber se os geógrafos e as instituições-chave aproveitarão esta oportunidade e verdadeiramente "atreve-se imaginar o imaginável" (Pezzani, 2010: 78). Esperamos que sim, porque, como notou Sylvia Wynter, no desafio de derrotar a colonialidade "a bola está conosco" (Wynter, 2003: 331).

\section{BIBLIOGRAFIA}

Daley, Patricia. Bob Geldof and the Livingstone connection: Africa not yet saved? Pambazuka News, n. 214, 2005. Disponível em: <http://www.pambazuka.org/governance/bob-geldof-andlivingstone-connection-africa-not-yet-saved>. Acesso em: 31 maio 2017.

Fanon, Frantz. Black skin, white masks. New York: Grove Press, 1967.

Gaztambide-Fernandez, Rubén. Decolonization and the pedagogy of solidarity. Decolonization: Indigeneity, Education \& Society, v. 1, 2012, p. 41-67.

Hooks, Bell. We real cool: Black men and masculinity. New York: Routledge, 2004.

Mahtani, Minelle. Toxic geographies: absences in critical race thought and practice in social and cultural geography. Social and Cultural Geography, v. 15, n. 4, 2014, p. 359-67.

Maldonaldo-Torres, Nelson. On the coloniality of being: contributions to the development of a concept. Cultural Studies, v. 21, n. 2-3, 2007, p. 240-70.

Mbembe, Achille. Decolonizing the university: new directions. Arts and Humanities in Higher Education, v. 15, n. 1, 2016, p. 29-45.

Mignolo, Walter. Racism as we sense it today. Publications of the Modern Languages Association of America, v. 123, n. 5, 2008, p. 1737-1742. 
Ndlovu-Gatsheni, Sabelo J. Decoloniality as the future of Africa. History Compass, v. 13, n. 10, 2015, p. 485-96.

Peake, Linda; Kobayashi, Audrey. Policies and practices for an antiracist geography at the millennium. The Professional Geographer, v. 54, n. 1, 2002, p. 50-61.

Pezzani, Lorenzo. Decolonizing architecture, or, 'How to inhabit your enemy's house?'. Contemporary Practices, v. VII, 2010, p. 72-9.

Quijano, Aníbal. Coloniality of power, Eurocentrism, and Latin America. Nepantla, v. 1, n. 3, 2000, p. 533-80.

RGS-IBG 2017 Chair's theme and plenary lectures. Disponível em: <http://www.rgs.org/WhatsOn/ ConferencesAndSeminars/Annual+International+Conference/Conference+theme.htm>. Acesso: 31 maio 2017.

Rivera Cusicanqui, Silvia. Ch'ixinakax utxiwa: a reflection on the practices and discourses of decolonization. South Atlantic Quarterly, v. 111, n. 1, 2012, p. 95-109.

Shilliam R 2015 Black Academia: the doors have been opened but the architecture remains the same. In: Alexander, Claire; Arday, Jason (eds.). Aiming higher: race, inequality and diversity in the academy. London: Runnymede, 2015, p. 32-35.

Sium, Aman; Desai, Chandni; Ritskes, Eric. Towards the 'tangible unknown': decolonization and the Indigenous future. Decolonization: Indigeneity, Education \& Society, v. 1, n. 1, 2012, p. I-XIII.

Tate, Shirley A. Racial affective economies, disalienation and 'race made ordinary'. Ethnic and Racial Studies, v. 37, n. 13, 2014, p. 2475-2490.

Tejeda, Carlos; Espinoza, Manuel; Gutierrez. Toward a decolonizing pedagogy: social justice reconsidered. In: Trifonas, Peter (ed.) Pedagogies of difference: rethinking education for social change London: Routledge, 2003, p. 10-40.

Todd, Zoe. An Indigenous feminist's take on the ontological turn: 'ontology' is just another word for colonialism. Journal of Historical Sociology, v. 29, n. 1, 2016, p. 4-22.

Todd, Zoe. Indigenous stories, knowledge, legal traditions, ontologies, epistemologies as unceded territory (or: hands off of our teachings), 2017. Disponível em: <https://zoestodd.com/2017/04/27/ indigenousstories-knowledge-legal-traditions-ontologies-epistemologiesas-unceded-territory-orhands-off-of-our-teachings/>. Acesso em: 31 maio 2017.

Tuck, Eve; Yang, K. Wayne. Decolonization is not a metaphor. Decolonization: Indigeneity, Education \& Society, v. 1, n. 1, 2012, p. 1-40.

Walter, Maggie; Butler, Kathy. Teaching race to teach Indigeneity. Journal of Sociology, v. 49, n. 3, 2013, p. 397-410.

Wynter, Sylvia. Unsettling the coloniality of being/power/truth/freedom: towards the human, after man, its overrepresentation - an argument. The New Centennial Review, v. 3, n. 2003, p. 257-337.

\section{RESUMOS}

O tema escolhido pela presidência da Conferência Anual da Royal Geographical Society (com o Institute of British Geographers) (RGS-IBG), de 2017, é "Descolonizando os conhecimentos geográficos: abrindo a geografia para o mundo". Este comentário explica porque é que essa busca de uma consciência crítica através do pensamento descolonial poderia fazer mais mal do que 
bem. Nós demostramos como a ênfase na descolonização dos conhecimentos geográficos mais do que estruturas, instituições e práxis reproduz colonialidade, porque recentra grupos pivilegiados, não indígenas e brancos na arquitetura global da produção de conhecimento. Argumenta-se que um movimento descolonial efetivo na geografia deve reconhecer a interseccionalidade da indigeneidade e raça, e é preciso que os termos com os quais a disciplina inicia debates sobre descolonização e descolonialidade sejam determinados por aqueles que são racializados como indígena e não brancos pela colonialidade.

The theme for the chair's plenaries at the 2017 Royal Geographical Society (with the Institute of British Geographers) (RGS-IBG) Annual Conference is 'Decolonising geographical knowledges: opening geography out to the world'. This commentary explains why this pursuit of critical consciousness via decolonial thinking could do more harm than good. We show how the emphasis on decolonising geographical knowledges rather than structures, institutions and praxis reproduces coloniality, because it recentres non-Indigenous, white and otherwise privileged groups in the global architecture of knowledge production. It is argued that an effective decolonial movement within geography must recognise the intersectionality of indigeneity and race, and necessitates that the terms on which the discipline starts debates about decolonisation and decoloniality are determined by those racialised as Indigenous and non-white by coloniality.

Le thème présidentielle de la conférence annuelle RGS-IBG 2017 s'intitule «Décolonisation des connaissances géographiques: l'ouverture de la géographie vers le monde ». Le commentaire suivant montre comment la recherche d'une conscience critique à partir de la pensée décoloniale peut être plus négative que positive. En soulignant la décolonisation des savoirs géographiques en detriment de la décolonisation des structures, des institutions et de la práxis en générale, ce mouvement continue à réproduire la colonialité dans la mesure où il repositionne les nonindigènes, les blancs et d'autres groupes privilégiés au centre de la production globale de la connaissance. Notre argument, c'est que l'effectivité de la décolonisation en géographie doit reconnaître l'interseccionalité entre l'indigéneité et la race, et que les termes du débat devront être déterminés par ceux qui sont racialisée par la colonialité du savoir tels que les autochtones et les non-blancs.

El tema elegido por el presidente de la Conferencia Anual de la Royal Geographical Society (con el Instituto de Geógrafos Británicos) (RGS-IBG), 2017, es "Descolonizando el conocimiento geográfico: abriendo la geografía al mundo". Este comentario explica por qué esta búsqueda de conciencia crítica a través del pensamiento descolonial podría hacer más daño que bien. Demostramos cómo el énfasis en la descolonización del conocimiento geográfico más que en estructuras, instituciones y praxis reproduce la colonialidad, porque reenfoca a grupos privilegiados, no indígenas y blancos en la arquitectura global de producción de conocimiento. Se argumenta que un movimiento descolonial efectivo en geografía debe reconocer la interseccionalidad de la indigeneidad y la raza, y los términos en los que la disciplina inicia los debates sobre la descolonización y la descolonialidad deben ser determinados por aquellos que son racializados como indígenas y no blancos por la colonialidad. 
ÍNDICE

Índice cronológico: Século XX

Palavras-chave: colonialidade, descolonização, conhecimento geográfico, indigeneidade, racismo, branquitude

Palabras claves: colonialidad, descolonización, indigenismo, racismo, blanquilidad

Keywords: coloniality, decolonisation, indigeneity, racism, whiteness

Mots-clés: colonialité, décolonisation, indigèneité, racism, blanquitude

Índice geográfico: Reino Unido

\section{AUTORES}

\section{JAMES ESSON}

Departamento de Geografia, Universidade Loughborough, Loughborough LE11 3TU. E-mail: j.esson@lboro.ac.uk

\section{PATRICIA NOXOLO}

Departamento de Geografia, Universidade de Birmingham, Birmingham B15 2TT. E-mail: p.e.p.noxolo@bham.ac.uk

\section{RICHARD BAXTER}

Escola de Geografia, Queen Mary Universidade de Londres, Londres E1 4NS. E-mail: richard.baxter@socsci.ox.ac.uk

\section{PATRICIA DALEY}

Escola de Geografia e Ambiente, Universidade de Oxford, Oxford OX1 3QY. E-mail: patricia.daley@ouce.ox.ac.uk

\section{MARGARET BYRON}

Departamento de Geografia, Universidade de Leicester, Leicester LE1 7RH. E-mail: mb416@le.ac.uk 